\title{
Livestock-associated methicillin-resistant Staphylococcus aureus responsible for human colonization and infection in an area of Italy with high density of pig farming
}

Monica Monaco ${ }^{* *}$, Palmino Pedroni ${ }^{2}$, Andrea Sanchini ${ }^{1,3,4}$, Annalisa Bonomini ${ }^{2}$, Annamaria Indelicato ${ }^{2}$ and Annalisa Pantosti ${ }^{1}$

\begin{abstract}
Background: Livestock-Associated MRSA (LA-MRSA) belonging to ST398 lineage, common among pigs and other animals, emerged in Central and Northern Europe, becoming a new risk factor for MRSA among farm workers. Strains belonging to ST398 can be responsible for human colonization and infection, mainly in areas with high livestock-farming. The aim of this study was to investigate the occurrence of livestock-associated methicillinresistant Staphylococcus aureus (LA-MRSA) human colonization and infections in an area of the Lombardy Region (Italy), the Italian region with the highest density of pig farming.

Methods: In the period March-April 2010, 879 nasal swabs were taken from subjects at admission to a local hospital serving an area of the Lombardy Region devoted to agriculture and farming. In the period March 2010February 2011, all MRSA strains from community-acquired infection (CAl) observed in the same hospital, were collected. Molecular characterization of the isolates included SCCmec typing, spa typing and multilocus sequence typing (MLST).

Results: Out of 879 nasal swabs examined, 9 (1\%) yielded MRSA. Five strains were assigned to sequence type (ST) 398 (spa t899, 3 isolates; t108 and t2922, 1 isolate each) and were therefore categorized as LA-MRSA. The other 4 isolates were likely of hospital origin. No strains were positive for Panton-Valentine Leukocidin genes. Twenty MRSA isolates were detected from CAl, 17 were from skin and soft-tissue infections and 3 from other infections. An MRSA isolate from otitis externa was t899/ST398 and PVL-negative, hence categorized as LA-MRSA. Four isolates were assigned to t127/ST1. Eight strains were PVL-positive community acquired (CA)-MRSA and belonged to different clones, the most frequent being ST8.
\end{abstract}

Conclusions: In an area of Italy with high density of pig farming, LA-MRSA is able to colonize the population and rarely to produce infections. Typical CA-MRSA is more common than LA-MRSA among CAI.

Keywords: Livestock-associated (LA)-MRSA, Colonization, Community-acquired infections, Pigs, ST398

\footnotetext{
* Correspondence: monica.monaco@iss.it

${ }^{1}$ Department of Infectious, Parasitic and Immuno-mediated Diseases, Istituto

Superiore di Sanità, viale Regina Elena, 299, Rome, Italy

Full list of author information is available at the end of the article
} 


\section{Background}

Methicillin-resistant Staphylococcus aureus (MRSA) emerged in hospital fifty years ago and swiftly became one of the most important hospital-associated (HA) pathogens being responsible for serious infections such as pneumonia and sepsis [1], and commonly characterized by a multi-drug resistant phenotype. Distinct lineages of HA-MRSA emerged in the years and became established with different success in different geographical locations [2].

Starting from the last decade, MRSA epidemiology largely changed, due to the emergence of new MRSA lineages, responsible for infections occurring in the community among patients without known risks factors for the acquisition of MRSA and without previous hospital contacts [3]. These strains, defined community acquired (CA)-MRSA, are mainly responsible for skin and softtissue infections (SSTI), although deep-seated infections such as necrotizing pneumonia, sepsis and meningitis, have also been reported $[3,4]$. CA-MRSA is characterized by the presence of the Panton-Valentine Leukocidin (PVL), a toxin that causes polymorphonuclear lysis and tissue necrosis [5] although its contribution to disease is still debated [6,7].

More recently, the emergence of an MRSA clone colonizing pigs and, more rarely, other farm animals (cattle and poultry) have been reported in Europe [8-10]. These strains, designated livestock-associated (LA)-MRSA and belonging to sequence type (ST)398, were mostly found in countries with high density of pig farming such as the Netherlands, Denmark and Germany [11,12]. These strains were able to colonize persons working in close contact with pigs, such as farmers and veterinarians. In Netherland and in Germany, $26.5 \%$ and $24 \%$ of pig farmers were colonized by LA-MRSA, respectively $[12,13]$. Wulf et al. found that $12.5 \%$ of veterinarians attending an international conference were colonized by MRSA of which $92.5 \%$ were LA-MRSA [14]. Farmers' family members, who are not in direct contact with pigs, were colonized at a lower frequency than the farm workers, indicating that inter-human transmission may occur [15].

Since LA-MRSA was found in dust from pig holdings [16], the environment could represent a vehicle for the transmission of LA-MRSA strains from animals to humans, allowing colonization of subjects who do not work directly with animals.

Infections due to LA-MRSA have been reported occasionally in farm workers and their family members [10]. In the Netherlands and Denmark where the percentage of HA-MRSA is very low $(<1 \%)$, LA-MRSA strains represent an important reservoir for human MRSA infections $[10,11]$. In the Netherland, by the end of 2008, LA-MRSA ST398 accounted for $42 \%$ of all new detected MRSA [12].

In Italy, LA-MRSA ST398 prevalence in farm workers is unknown. In the study previously cited, MRSA carriage in
Italian veterinarians was found to be $54 \%$ [14]. Two cases of serious infections due to LA-MRSA were described in Italian farmers in the Lombardy region: a man working on a pig farm who developed cellulitis and pyomiositis of the buttock and a man working on a dairy farm who developed necrotizing fasciitis of the neck $[17,18]$.

Other MRSA lineages different from ST398 were found in a European survey on the prevalence of MRSA in pig holdings. One lineage, characterized by spa type t127 and belonging to ST1 was predominantly reported from Italy [16], as shown also by other studies [19]. Franco et al. recently demonstrated that t127/ST1 isolates can be assigned to two genetically different clusters (porcine and human) and hypothesized that t127/ST1 strains could represent another lineage of LA-MRSA [20].

This study was undertaken to investigate the prevalence of LA-MRSA colonization and the occurrence of LA-MRSA infections in subjects living in an area of the Lombardy region, where livestock farming is a prominent activity.

\section{Methods}

\section{Setting of the study and source of isolates}

Two prospective studies were performed: a study on MRSA colonization in patients at hospital admission and a study on MRSA in community-acquired infections (CAI). Both studies were conducted at the Manerbio Hospital, a local hospital with 250 beds, serving a population of approximately 120.000 inhabitants. This area was chosen for the studies because a case of LA-MRSA infection was detected in 2008 [18], and because livestock farming is one of the main economical activities of this area, located in Lombardy, the region with the highest pig farming density in Italy (Figure 1) [21].

For the colonization study, in the period March-April 2010, all subjects presenting to the hospital for preadmission laboratory tests before planned surgery, were subjected to a nasal swab for MRSA screening. Swabs were plated onto Mannitol Salt agar and Brilliance MRSA agar plates (Thermo Fisher Scientific, Milan, Italy). No enrichment culture was performed. The isolates were identified using an automated system (Vitek2, bioMérieux, Marcy-l'Etoile, France) at the Manerbio Hospital laboratory. Interpretative criteria were those suggested by the Clinical and Laboratory Standards Institute (CLSI) [22].

The study on CAI was conducted from March 2010 to February 2011. The definition of CAI included infections in outpatients and in hospitalized patients, within $48 \mathrm{~h}$ from hospital admission, who had not been hospitalized in the previous 3 months. MRSA strains originating from CAI were collected and further characterized. Only the first MRSA isolate per patient was studied.

Nasal screening was part of the pre-admission routine tests for surgical patients at the hospital involved in the 


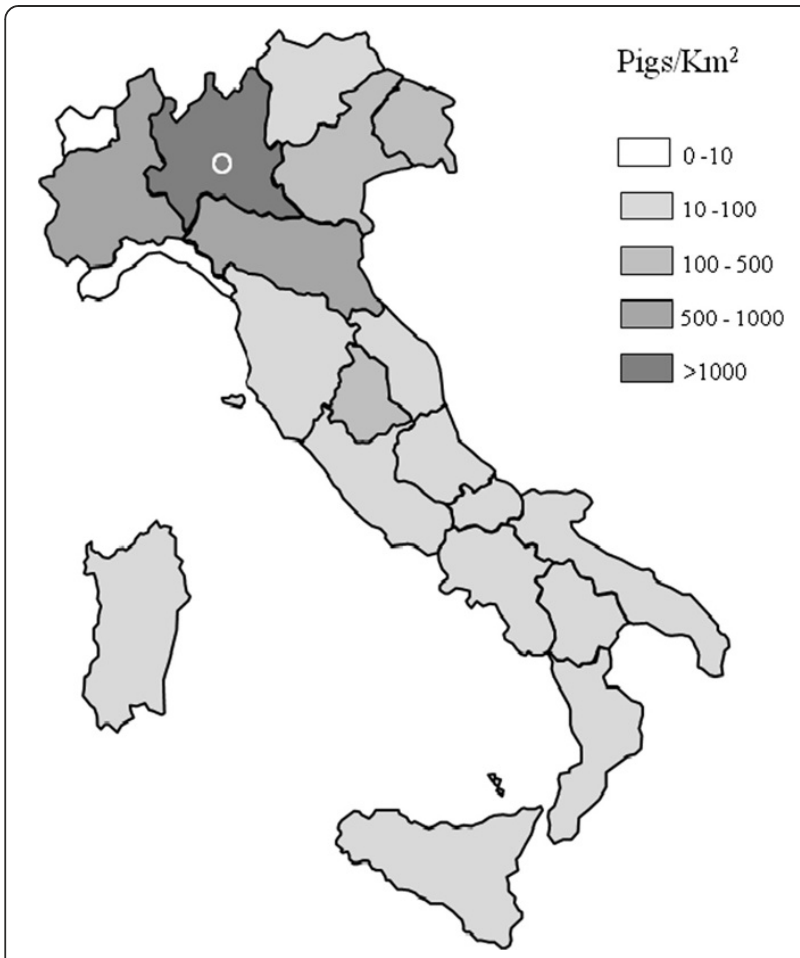

Figure 1 Density of breeding and fattening pigs in Italy. Adapted from [21]. The open circle indicates the area in the Lombardy Region where the study was performed.

study. Patients gave informed consent to the surgical procedure. Clinical data and samples (bacterial isolates) were anonymised before being used for the study. The study was exempt from Ethics Committee approval according to local and national regulations.

\section{Antimicrobial susceptibility testing}

In vitro susceptibility tests were obtained by an automated system (Vitek2, bioMérieux, Marcy-l'Etoile, France). The antimicrobial agents tested included: oxacillin, gentamicin, levofloxacin, erythromycin, clindamycin, tetracycline, rifampicin, vancomycin, teicoplanin, tigecycline, linezolid, trimethoprim-sulfamethoxazole, fusidic acid and mupirocin. Interpretative criteria were those suggested by the CLSI [22].

\section{Molecular characterization of the isolates}

Genomic DNA of MRSA isolates was extracted by the QIAamp DNA Mini Kit (QIAGEN, Hilden, Germany) and used as a template for polymerase chain reaction (PCR) amplification. S. aureus species and methicillin resistance status were confirmed by a duplex PCR targeting the genes nuc and mec. This PCR assay was developed in our laboratory by using primers published by Brakstad O.G et al., and Murakamy K., et al. [23,24]. The detection of the Panton-Valentine leukocidin (PVL) and of the arginine catabolic mobile element (ACME)specific $\operatorname{arcA}$ gene were performed as previously described [25]. The sak gene, encoding the plasminogen activator staphylokinase, was investigated on $\mathrm{t} 127$ strains by using published primers [26].

To determine the Staphylococcal chromosomal cassette mec (SCCmec) type, a combination of different multiplex PCR assays, discriminating the mec complex and the $\mathrm{ccr}$ genes complex, were applied by using protocols previously described [25]. Subtyping of the SCCmec type IV was obtained applying the method described by Milheiriço C., et al. [27].

Amplification, sequencing and analysis of the polymorphic region of the protein A gene (spa typing) was performed according to the standard protocol of Harmsen et al. [28]. MLST was performed on selected strains as described elsewhere [29]. The allelic profiles obtained were compared to those present in the MLST database.

\section{Results}

In the colonization study, 9 out of 879 patients (1\%) subjected to nasal screening prior to hospitalization were positive for MRSA. The age of patients carrying MRSA ranged from 17 to 71 years (median age 58); 4 patients were females and 5 were males. Out of the 9 patients colonized, 5 (56\%) carried LA-MRSA ST398. The overall LA-MRSA colonization rate was $0.56 \%$.

The characteristics of the MRSA isolates are shown in Table 1 . The 5 LA-MRSA strains were t899/SCCmec type IVa (2 isolates), t899/SCCmec IVc (1 isolate), t108/SCCmec type V ( 1 isolate) and t2922/SCCmec type V ( 1 isolate). The other isolates obtained from carriage belonged ST8 (3 isolates) and ST5 (1 isolate). None of the colonizing strains was positive for PVL genes.

In the infection study, 20 CAI due to MRSA were detected in the span of one year. Thirteen patients were females and 7 were males. The age of patients ranged from 8 months to 85 years (median age 53). Out of the 20 isolates, 17 were obtained from SSTI, 2 from otitis externa and 1 from bloodstream infection. One of the isolates from otitis externa was t899/ST398/SCCmec IVc and was categorized as LA-MRSA. Eight strains were positive for PVL and were considered typical CAMRSA. Of these, 3 were t008/ST8/SCCmec IVa and ACME-positive and therefore most likely corresponding to the USA300 clone. Another strain was t044/ST80/ SCCmec IVc and belonged to the European clone, while 3 other strains belonged to other lineages (ST22, ST772 and ST1).

ST8 and ST1 were found among both PVL-positive and PVL-negative isolates. PVL-negative ST8 were negative for ACME, too. All ST1 isolates (4 strains), belonged to spa type t127 and harboured the sak gene; of these one carried also PVL. The PVL-positive t127 isolate carried SCCmec V while the 3 PVL-negative t127 isolates carried SCCmec IVa. 
Table 1 Clinical informations, genotypic and phenotypic characteristics of MRSA responsible for human colonization and infections

\begin{tabular}{|c|c|c|c|c|c|c|c|c|c|c|}
\hline Isolate & $\begin{array}{l}\text { Patient } \\
\text { gender }\end{array}$ & $\begin{array}{c}\text { Patient } \\
\text { age (years) }\end{array}$ & $\begin{array}{c}\text { Type of } \\
\text { infection }\end{array}$ & $\mathrm{SCCmec}$ type & spa type & ST & $\mathrm{CC}$ & PVL & ACME & $\begin{array}{c}\text { Resistance pattern to } \\
\text { non beta-lactam antibiotics }\end{array}$ \\
\hline \multicolumn{11}{|c|}{ Colonization } \\
\hline Sau93 & M & 67 & - & IVC & t899 & 398 & 398 & - & - & ERY, CLI, SXT, TET \\
\hline Sau95 & $\mathrm{F}$ & 77 & - & $\mathrm{IVa}$ & t899 & 398 & 398 & - & - & ERY, CLI, TET \\
\hline Sau98 & $\mathrm{F}$ & 58 & - & $\mathrm{IVa}$ & t899 & 398 & 398 & - & - & TET \\
\hline Sau91 & M & 40 & - & V & $\mathrm{t} 108$ & 398 & 398 & - & - & LVX \#, TET \\
\hline Sau99 & M & 20 & - & V & t2922 & 398 & 398 & - & - & ERY, CLI, SXT, TET \\
\hline Sau96 & $\mathrm{F}$ & 61 & - & IVC & t008 & 8 & 8 & - & - & LVX, TET \\
\hline Sau97 & M & 66 & - & IVC & t008 & 8 & 8 & - & - & GN, LVX \\
\hline Sau92 & M & 71 & - & IVC & t008 & 8 & 8 & - & - & LVX \\
\hline Sau94 & $\mathrm{F}$ & 17 & - & V & t688 & 5 & 5 & - & - & ERY, CLI*, TET \\
\hline \multicolumn{11}{|l|}{ Infections } \\
\hline Sau150 & M & 18 & SSTI & $\mathrm{IVa}$ & t008 & 8 & 8 & + & + & LVX, ERY \\
\hline Sau116 & $\mathrm{F}$ & 71 & SSTI & $\mathrm{IVa}$ & t008 & 8 & 8 & + & + & LVX, ERY \\
\hline Sau161 & $\mathrm{F}$ & 27 & SSTI & $\mathrm{IVa}$ & t008 & 8 & 8 & + & + & LVX, ERY \\
\hline Sau85 & $\mathrm{F}$ & 46 & SSTI & $\mathrm{IVa}$ & $\mathrm{t} 121$ & 8 & 8 & + & - & LVX, ERY \\
\hline Sau86 & $\mathrm{F}$ & 35 & SSTI & IVc & t005 & 22 & 22 & + & - & ERY, CLI* \\
\hline Sau149 & M & 0.8 & SSTI & IVc & t044 & 80 & 80 & + & - & FUS, TET \\
\hline Sau151 & $\mathrm{F}$ & 2 & SSTI & V & $\mathrm{t} 127$ & 1 & 1 & + & - & GN, FUS, TET \\
\hline Sau119 & M & 38 & SSTI & V & t7445 & 772 & 1 & + & - & GN, ERY, CLI* SXT, \\
\hline Sau118 & $\mathrm{F}$ & 53 & SSTI & IVC & t008 & 8 & 8 & - & - & LVX \\
\hline Sau73 & $\mathrm{F}$ & 70 & SSTI & IVC & t008 & 8 & 8 & - & - & $\mathrm{LVX}^{\#}$, ERY, CLI* \\
\hline Sau159 & $\mathrm{F}$ & 85 & SSTI & IVC & t008 & 8 & 8 & - & - & LVX \\
\hline Sau163 & $\mathrm{F}$ & 53 & SSTI & IVc & t008 & 8 & 8 & - & - & GN, LVX, RIF \\
\hline Sau84 & M & 67 & SSTI & IVC & t008 & 8 & 8 & - & - & LVX, TET \\
\hline Sau121 & $\mathrm{F}$ & 83 & $\mathrm{BSI}$ & IVC & t008 & 8 & 8 & - & - & LVX \\
\hline Sau82 & $\mathrm{F}$ & 84 & SSTI & $\mathrm{IVa}$ & $\mathrm{t} 127$ & 1 & 1 & - & - & LVX, ERY, CLI* \\
\hline Sau83 & $\mathrm{F}$ & 9 & SSTI & $\mathrm{IVa}$ & $\mathrm{t} 127$ & 1 & 1 & - & - & ERY, CLI* , TET \\
\hline Sau162 & $\mathrm{F}$ & 61 & $\mathrm{OE}$ & $\mathrm{IVa}$ & $\mathrm{t} 127$ & 1 & 1 & - & - & GN, ERY, CLI*, TET \\
\hline Sau120 & M & 77 & SSTI & $\mathrm{IVh}$ & t515 & 22 & 22 & - & - & LVX \# \\
\hline Sau117 & M & 79 & SSTI & । & t041 & 228 & 5 & - & - & GN, LVX, ERY, CLI, RIF ${ }^{\#}$ \\
\hline Sau74 & M & 68 & $\mathrm{OE}$ & IVC & t899 & 398 & 398 & - & - & CLI, SXT, TET \\
\hline
\end{tabular}

$\mathrm{SCC}$ ec staphylococcal cassette chromosome mec, ST sequence type, CC clonal complex, PVL Panton-Valentine Leukocidin, ACME arginine catabolic mobile element.

${ }^{\S}$ GEN gentamicin, ERY erythromycin, CLI clindamycin, LVX levofloxacin, FUS fusidic acid, TET tetracycline, SXT co-trimoxazole, RIF rifampicin, * inducible clindamycin resistance; ${ }^{\text {intermediate resistance. }}$

SSTI skin and soft tissue infection, BSI bloodstream infection, OE otitis externa.

All the isolates, both from colonization and infections, were fully susceptible to vancomycin, teicoplanin, linezolid, tigecycline and mupirocin. All ST398 isolates were resistant to tetracycline. Resistance profiles to other non-beta-lactam antibiotics are shown in Table 1.

\section{Discussion}

In the present study we examined MRSA strains obtained from nasal screening of subjects prior to hospital admission and MRSA strains from CAI in an Italian Region, Lombardy, with the highest density of pig farming in Italy (48.2\% of all the pigs reared in the country) [21]. In particular, in the area where the study was conducted, a serious case of LA-MRSA infection was identified in 2008 [18].

Although the number of MRSA isolates obtained in this study was small, two different pictures emerged clearly: LA-MRSA, belonging to ST398, was prevalent (56\%) among the admission screening MRSA isolates, while, 
among the infections typical PVL-positive CA-MRSA predominate and only one strain belonging to ST398 was found. The percentage of LA-MRSA out of all MRSA colonizing strains is similar to that found in patients at admission to hospital in a region with high density of animal farming in Germany [30].

Unfortunately we were unable to obtain precise information about the working activity of the colonized subjects or of the infected patients, therefore we do not know if any of them worked or lived in close contact with pigs or other farm animals. The overall percentage of ST398 colonization in our study $(0.56 \%)$ is in the range (from $<0.01$ to $1.2 \%$ ) described in the Netherlands and Germany, regarding non-exposed healthy population $[12,13]$.

Our study confirms that the presence of LA-MRSA in human infections is rare. LA-MRSA isolates have been reported to sporadically cause mild or serious infections and a hospital outbreak $[17,18,31,32]$. According to data reported by Van Cleef et al., MRSA ST398 clone contributes only to a small fraction $(<2 \%)$ of all MRSA isolates in humans [9]. In a framework of an European survey conducted in 2011 on invasive infections caused by $S$. aureus predominant clones, we found that in Italy 1 out of 97 MRSA (1.5\%) strains belonged to a spa type indicative of ST398 (unpublished data), while in a previous survey carried out in 2006 no ST398 was found [2]. Therefore the ability of LA-MRSA to cause infections appears to be limited. This is probably due to the lack or rare occurrence of specific virulence factors, such as toxic shock syndrome toxic-1 (TSST-1) toxin and PVL, as shown by microarray and genomic studies [33,34]. In addition, LA-MRSA lacks the cluster of genes for humanspecific immune evasion encoded by Saф3 bacteriophage, and therefore is less virulent in humans than other staphylococcal clones [34,35].

In our study on outpatient infections, a prominent role was played by typical CA-MRSA strains. The presence of a variety of CA-MRSA clones including USA300, has been observed, as already reported in other studies $[4,25,36]$.

The recovery of isolates belonging to t127/ST1 is intriguing. t127/ST1 strains were recently isolated from pigs in Italy and the presence of a pig reservoir of this lineage has been hypothesized [20]. In the study by Franco et al., the $\mathrm{t} 127$ strains of porcine origin carried SCCmec type $\mathrm{V}$, while the $\mathrm{t} 127$ isolates of human origin harboured SCCmec type IVa and carried the sak gene, an immune-evasion factor present almost exclusively in human strains [20]. In our study three t127 strains carried SCCmec type IVa and the sak gene, indicating human origin. The only strain carrying SCCmec type V also harbored sak and PVL and therefore its porcine or human origin cannot be hypothesized.
Other clones causing infections are likely of hospital origin, such as the PVL-negative ST8 clone, resembling the "Lyon clone", a well adapted hospital clone that emerged in France and spread in other European countries, including Italy $[37,38]$. The presence of HA-MRSA in infections in the community might be due to previous contact of the patients with health-care settings.

\section{Conclusion}

Italy is one of the European countries with the highest MRSA rate according to data from the EARS-Net (around $40 \%$ ) and with the highest density of pig-livestock farming [16]. This is the first study evaluating the prevalence of LA-MRSA in the general population, in carriage and infections, in our country.

The presence of LA-MRSA in the general population confirms the importance of the animal reservoir and indicate the need for preventive measures to control the extent of LA-MRSA spread.

\section{Abbreviations}

MRSA: Methicillin-resistant Staphylococcus aureus; HA: Hospital-associated; HA-MRSA: Hospital-associated-MRSA; CA-MRSA: Community acquired-MRSA; PVL: Panton-Valentine leukocidin; LA-MRSA: Livestock-associated-MRSA; ST: Sequence type; CAl: Community-acquired infections; ACME: Arginine catabolic mobile element; SCCmec: Staphylococcal chromosomal cassette mec; MLST: Multilocus sequence typing; TSST-1: Toxic shock syndrome toxic1; EARS-Net: European Antimicrobial Resistance Surveillance-Network;

CC: Clonal complex; GEN: Gentamicin; ERY: Erythromycin; CLI: Clindamycin; LVX: Levofloxacin; FUS: Fusidic acid; TET: Tetracycline; SXT: Co-trimoxazole; RIF: Rifampicina; SSTI: Skin and soft tissue infection; BSI: Bloodstream infection; OE: Otitis externa.

\section{Competing interests}

The authors declare that they have no competing interests.

\section{Authors' contributions}

MM participated in the design of the study, carried out the molecular analyses and drafted the manuscript. PP, AB and Al participated in the design of the study and provided isolates. AS helped to perform the molecular typing. AP conceived the design of the study and helped to draft the manuscript. All authors read and approved the final manuscript.

\section{Acknowledgements}

We are grateful to F. Mancini for her help with SCCmec typing. This study was supported in part by a grant from the Italian Ministry of Health, CCM project: "Sorveglianza dell'antibiotico-resistenza in comunità, nelle infezioni trasmesse dagli alimenti e in quelle di origine zoonosica". The European Centre for Disease Prevention and Control has no responsibility for the views expressed in this article.

\section{Author details}

'Department of Infectious, Parasitic and Immuno-mediated Diseases, Istituto Superiore di Sanità, viale Regina Elena, 299, Rome, Italy. ${ }^{2}$ Presidio Ospedaliero Manerbio-Leno, Azienda Ospedaliera Desenzano del Garda, Lungomella Valsecchi, Brescia, Italy. ${ }^{3}$ Present address: Robert Koch-Institut (ZBS 1), Nordufer 20 D-13353 Berlin-Wedding, Berlin, Germany. ${ }^{4}$ Present address: European Public Health Microbiology Training Programme (EUPHEM), European Centre for Disease Prevention and Control (ECDC),

Tomtebodavägen 11a, 171 83, Stockholm, Sweden.

Received: 26 March 2013 Accepted: 23 May 2013

Published: 3 June 2013 


\section{References}

1. Pantosti A, Venditti M: What is MRSA? Eur Respir 2009, 34:1190-1196.

2. Grundmann $H$, Aanensen DM, van den Wijngaard CC, Spratt BG, Harmsen D, Friedrich AW: Geographic distribution of Staphylococcus aureus causing invasive infections in Europe: a molecular-epidemiological analysis. PLOS Med 2010, 7:e1000215.

3. Deleo FR, Otto M, Kreiswirth BN, Chambers HF: Community-associated meticillin-resistant Staphylococcus aureus. Lancet 2010, 375:1557-1568.

4. Sanchini A, Spitoni MG, Monaco M, Raglio A, Grigis A, Petro W, et al: Outbreak of skin and soft tissue infections in a hospital newborn nursery in Italy due to community-acquired meticillin-resistant Staphylococcus aureus USA300 clone. J Hosp Infect 2013, 83:36-40.

5. Boyle-Vavra S, Daum RS: Community-acquired methicillin-resistant Staphylococcus aureus: the role of Panton-Valentine leukocidin. Lab Invest 2007, 87:3-9.

6. Bubeck Wardenburg J, Bae T, Otto M, Deleo FR, Schneewind O: Poring over pores: alpha-hemolysin and Panton-Valentine leukocidin in Staphylococcus aureus pneumonia. Nat Med 2007, 13:1405-1406

7. Labandeira-Rey M, Couzon F, Boisset S, Brown EL, Bes M, Benito Y, Barbu EM, et al: Staphylococcus aureus Panton-Valentine leukocidin causes necrotizing pneumonia. Science 2007, 315:1130-1133.

8. Hasman H, Moodley A, Guardabassi L, Stegger M, Skov RL, Aarestrup FM: spa type distribution in Staphylococcus aureus originating from pigs, cattle and poultry. Vet Microbiol 2010, 141:326-331.

9. van Cleef BA, Monnet DL, Voss A, Krziwanek K, Allerberger F, Struelens M, et al: Livestock-associated methicillin-resistant Staphylococcus aureus in humans, Europe. Emerg Infect Dis 2011, 17:502-505.

10. Voss A, Loeffen F, Bakker J, Klaassen C, Wulf M: Methicillin-resistant Staphylococcus aureus in pig farming. Emerg Infect Dis 2005, 11:1965-1966.

11. Kock R, Harlizius J, Bressan N, Laerberg R, Wieler LH, Witte W, et al: Prevalence and molecular characteristics of methicillin-resistant Staphylococcus aureus (MRSA) among pigs on German farms and import of livestock-related MRSA into hospitals. Eur J Clin Microbiol Infect Dis 2009, 28:1375-1382

12. van Cleef BA, Verkade EJ, Wulf MW, Buiting AG, Voss A, Huijsdens XW, et al: Prevalence of livestock-associated MRSA in communities with high pig-densities in The Netherlands. PloS One 2010, 5:e9385.

13. Bisdorff B, Scholholter JL, Claussen K, Pulz M, Nowak D, Radon K: MRSAST398 in livestock farmers and neighbouring residents in a rural area in Germany. Epidemiol Infect 2012, 140:1800-1808.

14. Wulf MW, Sorum M, van Nes A, Skov R, Melchers WJ, Klaassen CH, et al: Prevalence of methicillin-resistant Staphylococcus aureus among veterinarians: an international study. Clin Microbiol Infect 2008, 14:29-34

15. Cuny C, Nathaus R, Layer F, Strommenger B, Altmann D, Witte W: Nasal colonization of humans with methicillin-resistant Staphylococcus aureus (MRSA) CC398 with and without exposure to pigs. PloS One 2009, 4:e6800.

16. European Food Safety Authority: Analysis of the baseline survey on the prevalence of methicillin-resistant Staphylococcus aureus (MRSA) in holdings with breeding pigs, in the EU, 2008. Part A: MRSA prevalence estimates. EFSA Journal 2009, 7:1376.

17. Pan A, Battisti A, Zoncada A, Bernieri F, Boldini M, Franco A, et al: Community-acquired methicillin-resistant Staphylococcus aureus ST398 infection, Italy. Emerg Infect Dis 2009, 15:845-847.

18. Soavi L, Stellini R, Signorini L, Antonini B, Pedroni P, Zanetti L, et al: Methicillin-resistant Staphylococcus aureus ST398, Italy. Emerg Infect Dis 2010, 16:346-348.

19. Battisti A, Franco A, Merialdi G, Hasman H, lurescia M, Lorenzetti R, et al: Heterogeneity among methicillin-resistant Staphylococcus aureus from Italian pig finishing holdings. Vet Microbiol 2010, 142:361-366.

20. Franco A, Hasman H, lurescia M, Lorenzetti $R$, Stegger $M$, Pantosti $A$, et al: Molecular characterization of spa type t127, sequence type 1 methicillinresistant Staphylococcus aureus from pigs. J Antimicrob Chemother 2011, 66:1231-1235

21. Bellini S, Alborali L, Massirio I, Cinotti S: Evoluzione del comparto suinicolo in Italia: criticità e fattori di rischio. L' Osservatorio 2010, 13:1-8.

22. Clinical and Laboratory Standards Institute: Performance Standards for Antimicrobial Susceptibility Testing: Eighteenth Informational Supplement M100-S18. Wayne, PA, USA: CLSI; 2008.

23. Brakstad OG, Aasbakk K, Maeland JA: Detection of Staphylococcus aureus by polymerase chain reaction amplification of the nuc gene. J Clin Microbiol 1992, 30:1654-1660.
24. Murakami K, Minamide W, Wada K, Nakamura E, Teraoka H, Watanabe S: Identification of methicillin-resistant strains of staphylococci by polymerase chain reaction. J Clin Microbiol 1991, 29:2240-2244.

25. Sanchini A, Campanile F, Monaco M, Cafiso V, Rasigade JP, Laurent F, Etienne J, et al: DNA microarray-based characterisation of Panton-Valentine leukocidinpositive community-acquired methicillin-resistant Staphylococcus aureus from Italy. Eur J Clin Microbiol Infect Dis 2011, 30:1399-1408.

26. van Wamel WJ, Rooijakkers SH, Ruyken $M$, van Kessel KP, van Strijp JA: The innate immune modulators staphylococcal complement inhibitor and chemotaxis inhibitory protein of Staphylococcus aureus are located on beta-hemolysin-converting bacteriophages. J Bacterio/ 2006, 188:1310-1315.

27. Milheirico C, Oliveira DC, de Lencastre H: Multiplex PCR strategy for subtyping the staphylococcal cassette chromosome mec type IV in methicillin-resistant Staphylococcus aureus: 'SCCmec IV multiplex'. J Antimicrobl Chemother 2007, 60:42-48.

28. Harmsen D, Claus H, Witte W, Rothganger J, Claus H, Turnwald D, et al: Typing of methicillin-resistant Staphylococcus aureus in a university hospital setting by using novel software for spa repeat determination and database management. J Clin Microbiol 2003, 41:5442-5448.

29. Enright MC, Day NP, Davies CE, Peacock SJ, Spratt BG: Multilocus sequence typing for characterization of methicillin-resistant and methicillin-susceptible clones of Staphylococcus aureus. J Clin Microbiol 2000, 38:1008-1015.

30. Kock R, Siam K, Al-Malat S, Christmann J, Schaumburg F, Becker K, et al: Characteristics of hospital patients colonized with livestock-associated meticillin-resistant Staphylococcus aureus (MRSA) CC398 versus other MRSA clones. J Hosp Infect 2011, 79:292-296.

31. van der Mee-Marquet N, Francois P, Domelier-Valentin AS, Coulomb F, Decreux C, Hombrock-Allet $C$, et al: Emergence of unusual bloodstream infections associated with pig-borne-like Staphylococcus aureus ST398 in France. Clin Infect Dis 2011, 52:152-153.

32. Wulf MW, Markestein A, van der Linden FT, Voss A, Klaassen C, Verduin CM: First outbreak of methicillin-resistant Staphylococcus aureus ST398 in a Dutch hospital, June 2007. Euro Surveill 2008, 13:pii: 8051.

33. Jamrozy DM, Fielder MD, Butaye P, Coldham NG: Comparative genotypic and phenotypic characterisation of methicillin-resistant Staphylococcus aureus ST398 isolated from animals and humans. PloS One 2012, 7:e40458.

34. Price LB, Stegger M, Hasman H, Aziz M, Larsen J, Andersen PS, Pearson T, Waters AE, Foster JT, Schupp J, et al: Staphylococcus aureus CC398: host adaptation and emergence of methicillin resistance in livestock. mBio 2012, 3:pii: e00305-11.

35. McCarthy AJ, Witney AA, Gould KA, Moodley A, Guardabassi L, Voss A, et al: The distribution of mobile genetic elements (MGEs) in MRSA CC398 is associated with both host and country. Genome Biol Evol 2011, 3:1 164-1174

36. Aschbacher R, Pichon B, Spoladore G, Pagani E, Innocenti P, Moroder L, et al: High clonal heterogeneity of Panton-Valentine leukocidin-positive meticillin-resistant Staphylococcus aureus strains from skin and soft-tissue infections in the Province of Bolzano, Northern Italy. Int J Antimicrob Agents 2012, 39:522-525.

37. Monaco M, Sanchini A, Grundmann H, Pantosti A: Vancomycin-heteroresistant phenotype in invasive methicillin-resistant Staphylococcus aureus isolates belonging to spa type 041. Eur J Clin Microbiol Infect Dis 2010, 29:771-777.

38. Dauwalder $\mathrm{O}$, Lina G, Durand G, Bes M, Meugnier H, Jarlier V, et al: Epidemiology of invasive methicillin-resistant Staphylococcus aureus clones collected in France in 2006 and 2007. J Clin Microbio/ 2008, 46:3454-3458.

doi:10.1186/1471-2334-13-258

Cite this article as: Monaco et al.: Livestock-associated methicillinresistant Staphylococcus aureus responsible for human colonization and infection in an area of Italy with high density of pig farming. BMC Infectious Diseases 2013 13:258. 